We start from the observation that the essentially singular function $u(r)=\exp \{-(\beta r+a / r)\}$, with $\beta, a>0$, satisfies the differential equation

$$
\begin{aligned}
-u^{\prime \prime}(r)+\left\{\left(a^{2} / r^{4}\right)-\left(2 a / r^{3}\right)-\left(2 a \beta / r^{2}\right)\right. & u(r) \\
& =-\beta^{2} u(r) .
\end{aligned}
$$

Thus $u(r)$ can be interpreted as $n \psi(r)$, where $\psi(r)$ is the (unnormalized) wave function for the lowest $s$ state in the potential

$$
U(r)=\left(a^{2} / r^{4}\right)-\left(2 a / r^{3}\right)-\left(2 a \beta / r^{2}\right),
$$

consisting of repulsive $r^{-4}$ and attractive $r^{-3}$ and $r^{-2}$ terms. More generally, and more usefully, the function

satisfies

$$
u(r)=\exp \left\{-\left[\beta r+(a / r)^{n}\right]\right\}
$$

$$
\begin{aligned}
-u^{\prime \prime}(r)+\left\{\frac{n^{2} a^{2 n}}{r^{2 n+2}}-\frac{n(n+1) a^{n}}{r^{n+2}}-\frac{2 n \beta a^{n}}{r^{n+1}}\right\} & u(r) \\
& =-\beta^{2} u(r),
\end{aligned}
$$

showing repulsive $r^{-(2 n+2)}$ and attractive $r^{-(n+2)}$ and $r^{-(n+1)}$ terms. ${ }^{1}$ For $n=5$ the potential is

$$
U(r)=\left(A / r^{12}\right)-\left(B / r^{7}\right)-\left(C / r^{6}\right),
$$

with $A, B, C>0$, which is similar to functions often used in analysis of molecular data. ${ }^{2}$

It seems clear, therefore, that a power-law repulsive core is conveniently discussed in terms of essentially singular wave functions, when the power is greater than 2. A potential function of the above kind can be expected to be more realistic than the often-used Morse potential, which is exponentially decreasing at the largest distances, in disagreement with expectations from the electrostatic van der Waals forces.

If the repulsive core in the interatomic interaction is stronger than any power law, a generalization of the present wave functions can be used

satisfies

$$
u(r)=\exp \{-[\beta r+f(r)]\}
$$

$$
-u^{\prime \prime}+\left\{\left(f^{\prime}\right)^{2}-f^{\prime \prime}+2 \beta f^{\prime}\right\} u=-\beta^{2} u .
$$

If $f(r)=A \exp (a / r)$ then

$$
\begin{aligned}
-u^{\prime \prime}+A \exp (a / r)\{[ & {[A \exp (a / r)-1]\left(a^{2} / r^{4}\right) } \\
- & \left.\left(2 a / r^{3}\right)-\left(2 \beta a / r^{2}\right)\right\} u=-\beta^{2} u,
\end{aligned}
$$

whose potential shows the very hard core

$$
U(r) \underset{r \rightarrow 0}{\rightarrow}(A / a)^{2}(a / r)^{4} \exp (2 a / r)
$$

while retaining a power-law asymptotic behavior.

Finally, a prescribed asymptotic power, say $r^{-6}$, can be built in, to agree with van der Waals expectations.

$$
u(r)=\exp \left(-\beta r-A \exp \left\{\frac{a}{r\left[1+(r / b)^{4}\right]}\right\}\right)
$$

satisfies the radial wave equation corresponding to a potential with a hard core as in (8) and with an attractive asymptotic leading term proportional to $r^{-6}$.

One's first reaction to the situation described by (10) might be that it must require very complicated algebra to explore a fit to experimental data. Note, however, that we are in possession of forms for both the groundstate wave function and the potential (obtained, as in previous cases, by differentiations of $u$ ). This is a big advantage. For instance, the equilibrium distance $r_{e}$ is to be interpreted as the value of $r$ for which $u$ is a maximum. The problem of generating excited state wave functions from the ground state appears to be a formidable one, on which we have no progress to report. Fits to vibrational data (and extraction of the magnitude of the leading asymptotic term) using these potentials for the molecules $\mathrm{N}_{2}$ and $\mathrm{I}_{2}$ are reported by one of us (A.C.) in a thesis. ${ }^{3}$

* Supported in part by the U. S. Atomic Energy Commission. $\dagger$ Now in Department of Physics, University of Wisconsin, Madison, Wisconsin.

${ }^{1}$ The special case $n=4$ and zero energy $(\beta=0)$ is used by F. Y. Wu and E. Feenberg, Phys. Rev. 122, 739 (1961).

${ }^{2}$ Y. P. Varshni, Revs. Modern Phys. 29, 664 (1957); E. R. Lippincott, R. Schroeder, and D. Steele, J. Chem. Phys. 34, 1448 (1961).

${ }^{3}$ A. Cohen, M.S. thesis, The Pennsylvania State University, December, 1961.

\section{Semiclassical Equivalence Relationship Applied to the Calculation of Molecular- Beam Scattering Phase Shifts}

\author{
RICHARD B. BERNSTEIN* \\ Physics Department, University College, London, England \\ and Chemistry Department, University of Michigan, Ann Arbor, \\ Michigan \\ (Received November 16, 1961)
}

A QUANTUM mechanical analysis of molecular beam scattering has been presented ${ }^{1}$; the results could be concisely represented within the semiclassical framework of Ford and Wheeler. ${ }^{2}$ In this note the semiclassical equivalence relationship is further exploited and applied directly to the phase shift calculation.

For a central potential of the form $V(r)=\epsilon f(r / \sigma)$, the Jeffreys-Langer-WKB approximation for the phase shift becomes, in terms of the reduced parameters of I

$$
\begin{aligned}
\eta^{*}\left(b^{*}, K\right)=\int_{x_{0}}^{\infty}\left[1-V^{*}(x) / K-\left(b^{*} / x\right)^{2}\right]^{\frac{1}{2}} d x \\
-\int_{b^{*}}^{\infty}\left[1-\left(b^{*} / x\right)^{2}\right]^{\frac{1}{3}} d x,
\end{aligned}
$$

where $\eta^{*}=\eta / A, b^{*}=b / \sigma=\left(l+\frac{1}{2}\right) / A, x=r / \sigma, A=k \sigma$, $k=\mu v / \hbar, K=E / \epsilon, V^{*}(x)=f(x)=V / \epsilon ; \epsilon, \sigma$ are potential parameters. 
Differentiating Eq. (1),

$\frac{d \eta^{*}}{d b^{*}}=-b^{*} J+b^{*} \int_{b^{*}}^{\infty} d x / x^{2}\left[1-\left(b^{*} / x\right)^{2}\right]^{1}=-b^{*} J+\pi / 2$,

where

$$
J \equiv \int_{x_{0}}^{\infty} d x / x^{2}\left[1-V^{*}(x) / K-\left(b^{*} / x\right)^{2}\right]^{\frac{1}{2}} .
$$

Comparison with the expression for the classical deflection function $\theta\left(b^{*}, K\right)=\pi-2 b^{*} J$ yields directly the equivalence relation $d \eta^{*} / \mathrm{d} b^{*}=\theta / 2$. Integration with the boundary condition

$$
\lim _{b^{*} \rightarrow \infty} \eta^{*}(K)=0
$$

yields

$\eta^{*}\left(b^{*}, K\right)=\frac{1}{2}\left[\int_{0}^{b *} \theta d b^{*}-\int_{0}^{\infty} \theta d b^{*}\right]=-\frac{1}{2} \int_{b^{*}}^{\infty} \theta d b^{*}$.

Starting with a purely classical calculation of the deflection function $\theta\left(b^{*}, K\right)$, one obtains directly the semiclassical reduced phases $\eta^{*}\left(b^{*}, K\right)$ and thus the phase shifts $\eta_{l}(k)$ and the quantum scattering cross sections.

Three examples are now considered: (1) rigid sphere, (2) inverse power, and (3) L-J $(12,6)$ potentials.

(1) Rigid-sphere potential. Classically, $\theta=2 \cos ^{-1} b^{*}$, $\left(0 \leq b^{*} \leq 1\right)$, independent of $K$, from which $\mathrm{Eq}$. (4) gives

$\eta^{*}\left(b^{*}\right)=b^{*} \cos ^{-1} b^{*}-\left(1-b^{* 2}\right)^{\frac{1}{2}}-\frac{\pi}{4 A}+\frac{1}{8 A^{2}}+O\left(\frac{1}{A^{3}}\right) ;$

$\eta_{l}=\left(l+\frac{1}{2}\right) \cos ^{-1}\left(\frac{l+\frac{1}{2}}{A}\right)-A\left[1-\left(\frac{l+\frac{1}{2}}{A}\right)^{2}\right]^{l}-\frac{\pi}{4}+\frac{1}{8 A}$.

Calculations were made for $A=30,20,10$, and 5 . For $l<\frac{2}{3} \mathrm{~A}$, Eq. (6) yielded $\eta_{l}$ differing by less than $\pm 0.02 \mathrm{rad}$ from the exact values (over a range of phases from -30 to $0 \mathrm{rad}$ ), the error increasing with $l / A$. Of course, the semiclassical treatment cannot yield phases for $l>A$.

(2) Inverse power potential. For illustration, consider $V=-C^{(6)} / r^{6}$. Classically, for small deflections, $\theta=-15 \pi C^{(6)} / 16 E b^{6}=-15 \pi / 4 K b^{* 6}$, from which $E \mathrm{q}$. (4) gives

$\eta^{*}\left(b^{*}, K\right)=3 \pi / 8 K b^{* 5} ; \quad \eta_{l}=3 \pi \hbar^{2} C^{(6)} k^{4} / 64 \mu\left(l+\frac{1}{2}\right)^{5}$.

This is identical with Massey and Mohr's ${ }^{3}$ result, the so-called "Jeffreys-Born" approximation.

(3) L-J $(12,6)$ potential. Deflection angles are available in tabular form ${ }^{4}$; thus simple graphical integration $[\mathrm{Eq}(4)]$ of $\theta\left(b^{*}, K\right)$ may be employed. From I it is noted that for $b^{*}>2, \eta^{*} \cong-3 \pi / 8 K b^{* 5}$ so that only over the region $b^{*}<2$ is numerical integration necessary. As an example, using the tabulated ${ }^{4} \theta\left(b^{*}, 4\right)$ values, 21 values of $\eta^{*}\left(b^{*}, 4\right)$ were calculated from Eq. (4), and found to differ from the exact values (cf. I) by less than \pm 0.0015 , corresponding to \pm 0.03 in $\eta_{l}$ (over a range of some 20 rads).

Direct application (i.e., graphical integration) of the JLWKB formula was also found to give results of similar accuracy $( \pm 0.03 \mathrm{rad})$, as expected, since the two methods are entirely equivalent. However, in the great many cases for which classical deflection functions are already available either in analytical or tabular form, the use of Eq. (4) gives results of good accuracy with a very minimum of effort.

It should be re-emphasized (see I) that the semiclassical treatment is totally inapplicable in the "bounded region" for $K<K_{0}$ ( $K_{0}$ is the critical value for classical orbiting) due to wave function penetration to the centrifugal barrier. In this region the full treatment ${ }^{1}$ involving integration of the radial wave equation is required.

The author appreciates the valuable discussions and kind hospitality of Professor Sir Harrie Massey (University College London).

* National Science Foundation Senior Post-doctoral Fellow, 1960-61.

${ }_{1}$ R. B. Bernstein, J. Chem. Phys. 33, 795 (1960), hereafter referred to as $I$.

${ }^{2}$ K. W. Ford and J. A. Wheeler, Ann. Phys. 7, 259, 287 (1959).

${ }^{3}$ H. S. W. Massey and C. B. O. Mohr, Proc. Roy. Soc. (London) A144, 188 (1934). See I for further discussion.

4 J. O. Hirschfelder, C. F. Curtiss, and R. B. Bird, Molecular Theory of Gases and Liquids (John Wiley \& Sons, Inc., New York, 1954).

\section{Equation of State of Liquids at Constant Volume}

\author{
E. B. SмIтн \\ Department of Physical Chemistry, Oxford University \\ Oxford, England \\ (Received October 23, 1961)
}

$I^{\mathrm{T}}$ $T$ was recognized at a very early stage in the experimental study of the equations of state of fluids that liquid isochores have a simple form. This view has been confirmed by recent experiments ${ }^{1,2}$ which showed that $\left(\partial^{2} P / \partial T^{2}\right)_{V}$ is zero within the experimental error over moderate ranges of pressure and that $(\partial P / \partial T)_{V}$ is a function of volume only. On the basis of these observations we may write the relation

$$
(\partial P / \partial T)_{V}=(R / V) f_{1}(V),
$$

which, in its integrated form, gives an equation of state of the type

$$
P V / R T=f_{1}(V)+\left[f_{2}(V) / T\right] .
$$

Recently, it has been shown that Eq. (2) can be applied to gases at high temperatures over a wide range of 